\title{
The role of food-cue exposure and negative affect in the experience of thought-shape fusion
}

Citation for published version (APA):

Coelho, J. S., Roefs, A. J., \& Jansen, A. T. M. (2010). The role of food-cue exposure and negative affect in the experience of thought-shape fusion. Journal of Behavior Therapy and Experimental Psychiatry, 41(4), 409-417. https://doi.org/10.1016/j.jbtep.2010.04.004

Document status and date:

Published: 01/12/2010

DOI:

10.1016/j.jbtep.2010.04.004

Document Version:

Publisher's PDF, also known as Version of record

Document license:

Taverne

Please check the document version of this publication:

- A submitted manuscript is the version of the article upon submission and before peer-review. There can be important differences between the submitted version and the official published version of record.

People interested in the research are advised to contact the author for the final version of the publication, or visit the DOI to the publisher's website.

- The final author version and the galley proof are versions of the publication after peer review.

- The final published version features the final layout of the paper including the volume, issue and page numbers.

Link to publication

\footnotetext{
General rights rights.

- You may freely distribute the URL identifying the publication in the public portal. please follow below link for the End User Agreement:

www.umlib.nl/taverne-license

Take down policy

If you believe that this document breaches copyright please contact us at:

repository@maastrichtuniversity.nl

providing details and we will investigate your claim.
}

Copyright and moral rights for the publications made accessible in the public portal are retained by the authors and/or other copyright owners and it is a condition of accessing publications that users recognise and abide by the legal requirements associated with these

- Users may download and print one copy of any publication from the public portal for the purpose of private study or research.

- You may not further distribute the material or use it for any profit-making activity or commercial gain

If the publication is distributed under the terms of Article $25 \mathrm{fa}$ of the Dutch Copyright Act, indicated by the "Taverne" license above, 


\title{
The role of food-cue exposure and negative affect in the experience of thought-shape fusion
}

\author{
Jennifer S. Coelho*, Anne Roefs, Anita Jansen \\ Department of Clinical Psychological Science, Faculty of Psychology and Neuroscience, Maastricht University, Maastricht, The Netherlands
}

\section{A R T I C L E I N F O}

\section{Article history:}

Received 28 October 2009

Received in revised form

19 April 2010

Accepted 27 April 2010

\section{Keywords:}

Thought-shape fusion

Cognitive distortions

Negative affect

Food cue

Dietary restraint

\begin{abstract}
A B S T R A C T
Thought-shape fusion (TSF) is a cognitive distortion that can be induced by imagining eating high-caloric foods, and involves increased guilt, feelings of fatness, and perceptions of weight gain and moral wrongdoing. Two studies were conducted to further elucidate this phenomenon. Study 1 investigated whether merely being exposed to fattening foods (without being asked to think about these foods) could induce a TSF-like experience. Study 2 investigated the relationship between negative affect and TSF-like experiences. The results suggested that TSF is specific to thinking about eating fattening foods, as mere exposure to high-caloric foods did not increase state TSF scores in healthy females relative to a neutral control condition. Furthermore, susceptibility to TSF is associated with negative affect. Healthy females with low levels of negative affect appear to be protected against TSF, medium negative affect is associated with susceptibility to TSF inductions, while those with high levels of negative affect appear to be particularly vulnerable to TSF-like experiences (even after imagining a neutral situation). Overall, the studies suggest that negative affect is associated with a TSF-like experience, and that TSF is a phenomenon that is experienced (to at least some extent) by females in the general population.
\end{abstract}

(c) 2010 Elsevier Ltd. All rights reserved.

\section{Introduction}

Food temptations are ubiquitous in today's society, with individuals regularly being confronted with a variety of food-related cues, including food advertisements, vending machines filled with high-caloric snacks, and a high-density of fast-food restaurants. There is evidence that exposure to food cues increases food-related thoughts, particularly in chronic dieters (Fedoroff, Polivy, \& Herman, 1997). Depending on the way in which individuals think about food, there may be profound cognitive and behavioral consequences of these thoughts. Research has indicated that thinking about eating fattening, high-caloric foods can induce feelings of guilt, fatness, and moral-wrong doing in healthy women without eating disorders (Coelho, Carter, McFarlane, \& Polivy, 2008). This phenomenon has been labeled as 'thought-shape fusion' (TSF; Shafran, Teachman, Kerry, \& Rachman, 1999), and is postulated to exist on both state and trait levels.

State TSF has been induced by asking people to imagine eating large quantities of fattening foods, and to write a sentence about eating these foods (e.g., Shafran et al., 1999). State TSF is associated

\footnotetext{
* Corresponding author. University of Savoie, Department of Psychology, Jacob Bellecombette BP 1104, 73011 Chambéry cedex, France. Tel.: +33 04797585 57; fax: +330479758591.

E-mail address: jennifer.coelho@univ-savoie.fr (J.S. Coelho).
}

with feelings of anxiety, guilt, fatness, perceived weight gain and/or moral wrong-doing. In contrast, trait TSF is measured by a selfreport questionnaire which assesses a general tendency towards TSF-related thoughts. Trait TSF is associated with eating pathology, and occurs to a greater extent in individuals with eating disorders (Shafran \& Robinson, 2004); however, a subset of healthy women also appear to experience a high degree of trait TSF (Shafran et al., 1999). In addition to the psychological factors associated with TSF (i.e., guilt, perceived weight gain, feelings of fatness), inducing state TSF can also lead to urges to neutralize the effects of imagining eating high-caloric foods, by either corrective behavior (e.g., crossing out the sentence about eating the foods, imagining exercising, or imagining eating a healthy food), or body checking to ensure that weight gain has not occurred (Radomsky, de Silva, Todd, Treasure, \& Murphy, 2002; Shafran et al., 1999).

Given that TSF is associated with eating pathology, Coelho et al. (2008) hypothesized that chronic dieters would exhibit a stronger response to a TSF induction (i.e., higher state TSF scores) than would non-dieters. However, their results indicated that restrained eaters did not exhibit as strong a response to the TSF induction as did unrestrained eaters. One speculation put forth by the authors was that chronic dieters were suppressing thoughts of food during the TSF induction. This postulation is supported by previous research indicating that dietary restraint is positively correlated with cognitive suppression (Nederkoorn \& Jansen, 2002). 
Therefore, in order to prevent such suppression, a more direct confrontation with food during the induction may be necessary to induce a TSF-like experience in chronic dieters.

In fact, direct confrontations with food cues in and of themselves may have similar psychological consequences as the TSF induction that has been used in previous studies (i.e., imagining eating fattening food and writing a sentence about eating this food). For example, attending to chocolates, chips and brownies led to a significant decrease in satisfaction with body weight in normalweight chronic dieters (Fett, Lattimore, Roefs, Geschwind, \& Jansen, 2009). Similarly, an interaction between dietary restraint and food-cue exposure has been found, with restrained eaters exhibiting a tendency to report lower satisfaction with their weight after exposure to high-caloric foods (Geschwind, Roefs, Lattimore, Fett, \& Jansen, 2008). If food-cue exposure leads to lower weight satisfaction, presumably it may also lead to behavioral effects consistent with avoidance of high-caloric foods. Indeed, Fishbach, Friedman, and Kruglanski (2003) demonstrated that exposure to highcaloric foods leads to intentions to avoid these foods, and choice of a low-caloric food (an apple) over a high-caloric food (chocolate bar). Therefore, food-cue exposure appears to produce both emotional/cognitive effects (i.e., lowered body satisfaction) as well as behavioral effects (i.e., healthier food choices).

The tendency to have poorer body image/weight satisfaction after being exposed to high-caloric foods also appears to extend to judgments about others, particularly in individuals with higher levels of eating pathology. For example, exposure to food cues led individuals with bulimia to rate another woman's body as larger (Carter, Bulik, Lawson, Sullivan, \& Wilson, 1996). Likewise, individuals who exhibited higher levels of eating pathology and also experienced higher levels of stress were more likely to judge others' bodies to be larger after food-cue exposure (Heilbrun \& Flodin, 1989). This line of research suggests that exposure to food leads to changes in body image (i.e., weight satisfaction), and perhaps even perceptual distortions of body weight/shape in both the self and others. This phenomenon appears to occur predominantly in chronic dieters. Given that food-cue exposure appears to influence body satisfaction and body-size judgments, it is possible that mere exposure to food-related cues (in the absence of a TSF induction) can lead to a TSF-like experience.

Consideration of factors that trigger body dissatisfaction also leads to an examination of the role of negative affect. Forbush and Watson (2006) suggest that emotional distress can be associated with increased feelings of fatness. Therefore, it is possible that TSF-like experiences (which include increased feelings of fatness) may also be linked with distress. In fact, it appears that these triggers for body dissatisfaction (i.e., food-cue exposure and negative affect) may interact. Recent research suggests that negative affect increases attentional bias for food cues in healthy females (Hepworth, Mogg, Brignell \& Bradley, 2010). Therefore, we might expect that individuals with negative affect are more likely to direct their attention to food-related cues (and subsequently experience a stronger TSF-like experience). As with food-cue exposure, negative affect also appears to have a main effect on body satisfaction and body-size ratings. Participants who were induced with a negative mood were more dissatisfied with their bodies than were those who were exposed to a positive mood induction (Taylor \& Cooper, 1992). Furthermore, those individuals who were concerned with their weight/shape were more likely to overestimate the size of their bodies after being induced with a negative mood than were non-weight-concerned individuals. The authors conclude that negative mood may lead to disturbances in body size perception. Similarly, individuals with bulimia rate their body size as larger after a negative mood induction (Carter et al., 1996).
Disturbances in body size perception and body dissatisfaction, both of which can be induced by negative affect, are part of a TSF-like experience. It is therefore possible that negative affect can influence this experience, firstly by its effects on attention to food-related cues (Hepworth et al., 2010), and secondly by its direct effects on body satisfaction and body-size ratings (Carter et al., 1996; Taylor \& Cooper, 1992). Some preliminary evidence for the postulation that negative affect is associated with TSF-like experiences comes from previous research demonstrating a link between depressive symptomatology and trait TSF scores (Shafran \& Robinson, 2004). While the relationship between TSF and eating pathology was significant even after controlling for depression, it remains possible that negative affect in and of itself may play a distinct role in TSF-like experiences.

Based on a review of the limited existing research on TSF, and the possible impact of food-cue exposure and negative affect on TSF-like experiences, three main questions have emerged. First of all, it is unclear whether chronic dieters in particular are somewhat unresponsive to TSF inductions (as in Coelho et al., 2008), or whether they would be responsive if the induction would include a more direct (e.g., visual) exposure (as in Fett et al., 2009). Secondly, it has not yet been tested whether mere exposure to cues for high-caloric foods (in the absence of instructions to imagine eating these foods) can induce a TSF-like experience in healthy individuals without eating disorders. Given that chronic dieters exhibit changes in weight satisfaction (Fett et al., 2009; Geschwind et al., 2008) after exposure to high-caloric foods, it is conceivable that mere exposure can lead to features of state TSF (e.g., guilt, feelings of fatness). Finally, the relationship between negative affect and TSF has not yet been established. Based on the findings of Carter et al. (1996), who demonstrated that negative affect led to larger body-size ratings, we expected that negative mood inductions could lead to a heightened TSF response. The current studies were designed in order to test these questions, and investigate the effects of manipulating both the manner of TSF induction and affect on TSF-like experiences.

\subsection{Study 1: hypotheses}

It was expected that both TSF induction and mere food-cue exposure would induce higher levels of state TSF (e.g., guilt, anxiety, feelings of fatness, and perceived weight gain and moral wrongdoing) than would a neutral control induction. We predicted that by using a modified TSF induction, which included a direct food-cue exposure, chronic dieters would be more responsive than would non-dieters. Furthermore, based on previous research demonstrating that exposure to high-caloric foods leads to an increased likelihood of choosing a healthy food versus an unhealthy option (Fishbach et al., 2003), it was expected that both TSF induction and mere exposure to food-related cues would lead to a higher likelihood of participants choosing a healthy food (versus an unhealthy food) relative to those in a control condition. However, it was expected that only the TSF induction, but not mere exposure to high-caloric foods, would lead to higher negative affect and more neutralization behavior. This hypothesis was based on previous research demonstrating that mere food-cue exposure does not lead to changes in affect (e.g., Coelho, Jansen, Roefs, \& Nederkoorn, 2009), while exposure to a TSF induction does increase negative affect (Coelho et al., 2008).

\section{Method}

\subsection{Participants}

A total of 68 female undergraduates participated in exchange for either partial psychology course credit or a voucher for $€ 7.50$. The 
mean age of participants was 20.5 years $(S D=2.6)$, and their mean body mass index was $23.3(\mathrm{SD}=4.5)$.

\subsection{Measures}

Participants were provided with Dutch translations of all questionnaires.

\subsubsection{Positive and negative affect schedule (PANAS; Watson, Clark, Er Tellegen, 1988)}

The PANAS is a 20 -item questionnaire which has been demonstrated to be a valid and reliable tool to assess positive and negative affect, with high Cronbach's alpha for both the positive and negative affect subscales (.89 and .85 respectively, Watson et al., 1988). The Cronbach's alphas obtained in this research were .81 (positive affect subscale) and .80 (negative affect subscale). Participants were instructed to rate their mood "right now, at this moment" when completing this questionnaire.

\subsubsection{State thought-shape fusion scale (state TSF; adapted from Radomsky et al., 2002)}

A 7-item questionnaire was developed to assess aspects of thought-shape fusion, including: feelings of anxiety and guilt, the likelihood that they had gained weight from imagining the situation/writing the sentence, how much fatter they felt after imagining the situation/writing the sentence, how much they desired a high-caloric food, the extent to which imagining the situation/ writing the sentence was morally wrong, and the extent to which they wanted to reduce the effects of imagining the situation/ writing the sentence. The questions were worded in such a way that the same questionnaire was provided to all individuals, regardless of their assigned condition. Participants were asked to rate each of the items on a scale of 0 (not at all) to 100 (completely), and the scores on each of the items were summed to create a total score (giving a possible range of scores on this measure from 0 to 700). The obtained Cronbach's alpha on this 7-item scale was .69.

\subsubsection{Trait thought-shape fusion scale (Trait TSF; Shafran et al., 1999)}

This is a 34-item questionnaire used to assess general tendencies towards TSF, with participants rating their responses to each question on a 5-point scale, with 0 representing 'not at all', and 4 representing 'totally'. A total score is obtained by adding together all items, with higher scores demonstrating a higher degree of TSF. This questionnaire is unidimensional, with high internal consistency (Shafran et al., 1999). The obtained Cronbach's alpha on this 34-item scale was .96 .

\subsubsection{Restraint scale (Polivy, Herman, E Howard, 1988)}

The Restraint Scale is an 11-item questionnaire which assesses concern with dieting and weight fluctuations (the score is based on summing items 1 through 9 and item 11 ; item 10 is not scored, but is used to prompt participants to indicate the discrepancy between their highest weight ever and their ideal weight). Higher scores are indicative of higher weight- and shape-related concerns. This measure has good internal consistency (Cronbach's alpha $=.82$ ) and test-retest reliability (Allison, Kalinsky, \& Gorman, 1992). The Cronbach's alpha obtained in this study was .83 .

A median split is typically performed on restraint scores in order to identify individuals as either restrained eaters (i.e., chronic dieters) or unrestrained eaters (i.e., non-dieters). In accordance with previous studies (e.g., Coelho et al., 2008), participants with a score of 14 or above were classified as restrained eaters $(n=34)$, while those with a score below 14 were classified as unrestrained eaters $(n=34)$.

\subsection{Procedure}

Participants were recruited to participate in a study which was purportedly testing some new measures for studies on personality, and were informed that they would imagine different situations, and fill out some general questionnaires about themselves. During scheduling, participants were asked to report their height and weight (this question was embedded among other demographic questions, including age and program of study). Upon arrival for the experimental session each participant completed the informed consent form, and was asked to rate her current mood on the PANAS. The experimenter then asked the participant to imagine one of three situations, depending on the condition to which she had been randomly assigned. The description of each of the conditions is below.

\subsubsection{Control}

Participants were asked to think about a book that they had read or a movie they had seen recently, and were then asked to imagine in vivid detail chatting about this book or movie with a friend. Participants were then asked to write down the sentence "I am chatting about ___ (inserting the name of the book or movie that they were imagining chatting about; as in Coelho et al., 2008). Throughout the experimental session, a tray of office supplies was present on a table adjacent to the participant.

\subsubsection{Mere food-cue exposure}

As in the control condition, participants were asked to imagine chatting about a book or movie, and to write down the sentence "I am chatting about____ (inserting the name of the book or movie that they were imagining). Throughout the experimental session, a tray filled with high-caloric foods (including chocolate bars, potato chips, candies, coated peanuts, and licorice) was present on a table adjacent to (and within view of) participants.

\subsubsection{Thought-shape fusion}

As in the mere exposure condition, a tray filled with high-caloric foods was present on a table adjacent to participants. When the time for the induction arrived, this tray was placed in front of participants, and they were asked to choose one of the foods on the tray that they particularly liked. Participants were then asked to imagine in vivid detail that they were eating large quantities of this food, and were prompted to imagine the taste, look, and smell of this food. Participants were then asked to write down the sentence "I am eating " (inserting the name of the food they were imagining).

After the induction, participants were asked to complete the PANAS and the State TSF questionnaire. Participants were also asked to estimate their current weight, and were informed that if they were accurate in their estimation, they would receive a small reward at the end of the study. This self-reported weight was compared with the self-report obtained prior to participation, to assess for differences. Participants were then given an opportunity to neutralize the induction. The experimenter explained that sometimes participants felt a desire to reduce the effects of having written the sentence/imagined the situation, and were prompted by the experimenter that if they desired, they could cross out the sentence, or write down something else. The experimenter took note of whether participants chose to neutralize the statement.

Finally, participants completed the Restraint Scale. The experimenter measured participants' height and weight, and informed them that everyone received a small reward to express gratitude for their participation (regardless of the accuracy of their weight estimate), and were given a choice of an apple, chocolate bar, or $€ 1$. The experimenter recorded participants' choice, and provided 
participants with this choice along with their compensation for participation (i.e., the voucher or participation point). Participants received a debriefing form upon completion of the study. All procedures were approved by the ethical committee at the university where this research was conducted.

\section{Results}

\subsection{State TSF}

An ANOVA was conducted in order to assess the 'state' TSF-like experience, with the total State TSF score entered as a dependent variable, and the induction condition (TSF, mere exposure, vs. control) and restraint status (restrained vs. unrestrained) entered as independent variables. A significant main effect of condition emerged $\left(F_{2,62}=4.86, p<.02\right)$, with Bonferroni-corrected pairwise comparisons demonstrating that those in the TSF induction reported significantly higher State TSF scores $(M=130.1, \mathrm{SD}=17.1)$ than did either those in the mere exposure $(M=69.1, \mathrm{SD}=16.7)$ or control conditions $(M=62.0, \mathrm{SD}=16.7$; both $p$ 's $<.05)$. No significant effect of restraint status emerged, nor was there an interaction between restraint and condition $\left(F^{\prime} s<1\right)$.

In order to determine whether participants exposed to the TSF induction actually believed that they had gained weight as a result of imagining the food, participants' self-reported weight postinduction was compared with their self-reported weight at scheduling. However, no significant main effects emerged, nor was there an interaction between restraint and condition (all $F^{\prime} s<1$ ).

\subsection{Negative affect}

A repeated-measures ANOVA was conducted on pre- and postinduction negative affect scores from the PANAS. However, no effects of time on negative affect emerged, nor were there any interactions between the independent variables (all $F^{\prime} s<1$ ). Those in the TSF induction condition did not exhibit an increase in negative affect post-induction; however, it remained possible that a relationship existed between negative affect and state TSF. Indeed, a correlational analysis demonstrated a significant positive correlation between post-induction negative affect and State TSF scores $(r(68)=.314, p<.01)$.

\subsection{Neutralization}

Chi-square analyses were performed to determine whether there was a significant effect of induction condition on the likelihood of neutralization. Likelihood ratio chi square statistics were used to assess whether the results were significant, as this statistic is less affected by small expected frequencies than is the Pearson chi square (Hays, 1963). A significant effect of condition on neutralization emerged $\left[\mathrm{L}^{2}(2, \mathrm{~N}=68)=6.64, p \leq .04\right]$. Follow-up analyses indicated that participants in the TSF induction condition were significantly more likely to neutralize (with $18 \%$ of participants neutralizing) than were participants in the control condition $\left(0 \%, \mathrm{~L}^{2}(1, \mathrm{~N}=45)=6.13, p \leq .02\right)$. The mere exposure condition ( $4 \%$ neutralized) did not differ significantly from either the control or TSF induction.

Follow-up analyses demonstrated that it was exclusively restrained eaters who neutralized after the TSF induction [ with 36\% of restrained eaters in the TSF condition neutralizing, $\mathrm{L}^{2}(2, \mathrm{~N}=34)=10.21$, $p \leq .01$, and that there were no significant differences across conditions for unrestrained eaters $\left[\mathrm{L}^{2}(2, \mathrm{~N}=34)=2.32, p>.3 ; 0 \%\right.$ neutralized in the TSF condition].

\subsection{Gift choice}

No significant effects of condition emerged on participants' choice of gift $\left[\mathrm{L}^{2}(4, \mathrm{~N}=68)=1.50, p>.8\right]$. The majority (59\%) chose $€ 1$ as their parting gift, while $31 \%$ chose an apple and $10 \%$ chose a chocolate bar.

\subsection{Trait TSF}

There were no main effects of induction condition on Trait TSF scores $\left(F_{1,60}=.38, p>.6\right)$, supporting the trait nature of this measure. There was a significant effect of dietary restraint on Trait TSF scores $\left(F_{160}=27.44, p \leq .001\right)$, with restrained eaters reporting higher scores $(M=32.4, \mathrm{SD}=21.9)$ than did unrestrained eaters $(M=10.3, \mathrm{SD}=9.3)$. The interaction between condition and restraint did not reach significance $\left(F_{2,60}=2.02, p \leq .14\right)$.

To assess whether a relationship exists between State and Trait TSF scores, correlational analyses were performed separately for each of the induction conditions (with a Bonferroni correction, $p<.016)$. A significant correlation between State and Trait TSF scores was obtained only for participants in the TSF induction condition ( $\mathrm{r}(20)=.536, p \leq .015)$; the correlations for those in the mere exposure $(\mathrm{r}(23)=.415, p \leq .049)$ and control conditions $(r(23)=.337, p \leq .116)$ did not reach statistical significance after the Bonferroni correction (perhaps due to a lack of power).

\section{Discussion}

As expected, the TSF induction led to an increase in the experience of state TSF, and increased neutralization behavior, relative to a control induction. However, contrary to the hypotheses, both restrained and unrestrained eaters were responsive to the modified TSF induction, and exhibited approximately equal levels of state TSF. Providing some support for the original hypothesis that restrained eaters would be more susceptible to TSF, however, was the finding that only restrained eaters exhibited neutralizing behavior after being induced with TSF. This neutralizing behavior suggests that the induction may have been perceived as somewhat aversive; however, given the lack of interaction between TSF induction and restraint status on state TSF scores, and the fact that restrained eaters did not report higher negative affect after TSF induction, there is no direct support for this postulation. Providing further support for the fact that restrained eaters may be more prone to TSF-like experiences is the finding that they have elevated scores on the Trait TSF scale relative to unrestrained eaters, a finding which is consistent with previous research (Coelho et al., 2008).

While it was originally hypothesized that mere exposure to high-caloric foods could induce some degree of state TSF, there were in fact no differences in state TSF scores between the mere exposure and control conditions. This finding supports the notion that there is something specific about imagining eating the highcaloric foods inherent in the TSF induction which leads to a TSF-like experience (Coelho et al., 2008). The fact that both restrained and unrestrained eaters responded to the TSF induction suggests that there may be some powerful effects of food-cue exposure in the context of a TSF induction. The previous study of Coelho et al.s on the effects of TSF induction failed to induce a TSF-like experience in restrained eaters relative to a control condition, while the current study (which exposed participants to a variety of high-caloric foods during the TSF induction) did demonstrate increased TSF in restrained (and unrestrained) eaters. That being said, it is important to note that the mere exposure condition included distraction during the exposure period - participants were not directed to focus their attention on the foods, and were occupied with 
completing a variety of questionnaires and imagining a neutral, non-food-related situation during the presence of the food. To conclude more definitively that mere food-cue exposure has no effects on state TSF, the effects of mere exposure to food cues in the absence of distraction should be tested in future research.

It was initially expected that food-cue exposure and TSF induction would lead to a higher proportion of participants choosing an apple (over a chocolate bar or $€ 1$ ); however, there were no effects of either the TSF induction or mere exposure condition on gift choice. It appears that any possible effects that may have emerged were obscured by the inclusion of $€ 1$ as one of the gift choices, with the majority of participants choosing this option. The decision to include the $€ 1$ option was to allow a nonfood-related reward; however, given that this led to apparent floor effects of the other choices, a forced-choice option between a 'healthy' and 'unhealthy' food, as in Fishbach et al. (2003), may be preferable.

Finally, it was initially expected that the TSF induction would lead to increased negative affect. Contrary to both the hypothesis and previous research (Coelho et al., 2008), there were no increases in negative affect after the TSF induction. One limitation of the current study was the use of a modified TSF induction, in which participants were instructed to choose a food from a limited selection in front of them. It is possible that this modified procedure, in contrast to the standard induction in which individuals choose the food to imagine, may have dampened the response to the TSF induction. However, the fact that significant effects on state TSF scores emerged using this modified induction supports the power of the modified induction. Furthermore, the fact that a significant positive correlation between negative affect and state TSF scores emerged suggests that there is some relationship between negative affect and state TSF. This is consistent with previous research demonstrating a correlation between depression and trait TSF (Shafran \& Robinson, 2004).

A further objective of the current research was to establish whether negative affect can influence TSF-like experiences. Previous research has established that negative mood inductions lead to increased body dissatisfaction and body-size ratings (e.g., Carter et al., 1996; Taylor \& Cooper, 1992). Given that inducing negative affect has harmful effects on self-image, it is possible that by experimentally decreasing negative affect (e.g., by giving a positive mood induction), body dissatisfaction can be decreased. However, the existing research is somewhat ambiguous in terms of the interpretation of positive mood inductions. Taylor and Cooper did not include a control group in their study, but only a positive and negative mood induction; therefore, it is not clear whether positive mood inductions can be protective against body dissatisfaction (relative to a control condition). Some evidence for the notion that positive mood may be protective against body dissatisfaction has been obtained from research in a population of individuals with bulimia, who reduced estimates of their body size after receiving a positive mood induction (Kulbartz-Klatt, Florin, \& Pook, 1999). We designed a second study to further investigate the relationship between mood and TSF, and to determine whether reductions in negative mood can be protective against state TSF in non-eating-disordered individuals.

\section{Study 2}

In order to determine whether mood influences susceptibility to a TSF induction, a 3 (mood: positive, neutral, negative) $\times 2$ (TSF vs. control induction) between-subjects design was used. It was expected that the TSF induction would lead to higher state TSF scores, and a higher likelihood of neutralizing behavior than would the control induction; however, it was expected that mood would interact with the induction, such that those who were exposed to a negative mood induction would have stronger responses to the TSF induction, and that those exposed to a positive mood induction would have weaker responses to the TSF induction (compared to those exposed to a neutral mood induction). It was also expected that the TSF induction would influence food choice, such that those exposed to the TSF induction would be more likely to choose a healthy food than would controls. In order to address the limitations of Study 1 (i.e., the possible floor effects as a result of including the choice of $€ 1$ ), the gift choice was limited to either a healthy or unhealthy food. Because there were no main effects of restraint status on state TSF in Study 1 (nor did dietary restraint interact with the TSF induction condition), restraint status was not included as an independent variable for the current study (though its measurement was included).

In Study 1, TSF was induced by presenting participants with a tray of high-caloric foods and asking them to imagine eating large quantities of one of these foods. This approach had the advantage of providing a visual cue to participants, which could perhaps enhance their experience of the induction (especially in restrained eaters, who may attempt to suppress food-related thoughts). However, one disadvantage of this modified induction is that it limits participants' choice of food to something present on the tray. Previous studies using the standard TSF induction (e.g., Coelho et al., 2008; Shafran et al., 1999) allowed participants to imagine any type of high-caloric food that they find to be forbidden. Pilot studies in our laboratory, as well as previous research (Coelho et al., 2008) demonstrated that many participants choose to imagine savory high-caloric foods (e.g., pizza, French fries, or hamburgers), which were not represented in the modified TSF induction. Additional limitations of this modified induction include the fact that the foods identified as 'forbidden' can differ depending on an individual's dietary restraint status (Gonzalez \& Vitousek, 2004). Furthermore, studies investigating the effects of food-cue exposure typically allow individuals to identify their preferred foods, presumably because this can enhance the manipulation (e.g., Jansen, Broekmate, \& Heymans, 1992; Legenbauer, Voëgle, \& Rüddel, 2004; Soetens, Braet, van Vlierberghe, \& Roets, 2008).

Consequently, it may be more powerful to employ an induction in which participants can identify their own forbidden food. Finally, negative affect may be associated with desire for different kinds of foods across individuals, ranging from chocolates and sweets, to savory foods (Lafay et al., 2001). Given the plan to manipulate negative affect in the current study, it heightened concerns about the limitations of the modified TSF induction, in its restriction of the foods participants could imagine. Therefore, the decision was made to use the original, unmodified TSF induction in Study 2.

\section{Method}

\subsection{Participants}

A total of 72 female undergraduates participated in exchange for either partial psychology course credit or a voucher for $€ 7.50$. The mean age of participants was 21.3 years $(S D=3.4)$, and their mean body mass index was $22.3(\mathrm{SD}=2.8)$. A total of 32 individuals were identified as restrained eaters (i.e., a Dietary Restraint score of 14 or above), while 40 individuals were identified as unrestrained eaters.

\subsection{Measures}

As in Study 1, participants were provided with Dutch translations of the PANAS (Cronbach's alpha $=.77$ and .81 for the positive and negative affect subscales respectively), State TSF Scale 
(Cronbach's alpha $=.71)$, Trait TSF Scale (Cronbach's alpha $=.96$ ), and the Restraint Scale (Cronbach's alpha $=.77$ ).

\subsection{Procedure}

As in Study 1, participants were recruited to participate in a study which was purportedly testing new measures for studies on personality. Participants reported their height and weight (among other demographic information) during scheduling. Once at the laboratory, participants completed the informed consent form, and were provided with the PANAS and asked to rate their current mood. The experimenter then asked participants to watch one of three film clips, depending on the mood condition to which the participant had been randomly assigned. Participants in the negative mood induction watched a clip from The Champ (2 m $44 \mathrm{~s}$ ) in which a young boy watches his father die, while those in the positive mood induction watched a clip from When Harry Met Sally ( $2 \mathrm{~m} 52 \mathrm{~s}$ ) in which the characters discuss female orgasm. These films have been shown to reliably induce sadness and amusement, respectively (Gross \& Levenson, 1995). Participants in the neutral mood induction watched a dialogue-free clip about glass-blowing (2 m $50 \mathrm{~s})$.

After watching the film clip, participants completed the PANAS again as a manipulation check. Then, participants received either a control or TSF induction (depending on the condition to which they had been randomly assigned). As in Study 1, participants in the control induction were asked to imagine chatting about a book or movie with a friend, and to write down the sentence "I am chatting about

". Those in the TSF condition were asked to think of a food that they would consider to be particularly fattening, or high in calories, and to imagine in vivid detail that they were eating large quantities of this food. As in Study 1, they were prompted to imagine the taste, look, and smell of this food, and were also asked to write down the sentence "I am eating

name of the food they were imagining). " (inserting the

Participants then completed the State TSF questionnaire, and their estimate of their current weight. As in Study 1, they were informed that if they were accurate in their estimation, they would receive a small reward at the end of the study. Participants were then given an opportunity to neutralize the sentence that they had written. Finally, participants were provided with the Restraint Scale. The experimenter informed participants that everyone received a small reward to express gratitude for their participation (regardless of the accuracy of their weight estimate), and were given a choice of either an apple or a chocolate bar. The experimenter recorded participants' choice, and then measured their height and weight. Participants received a debriefing form upon completion of the study. The procedures were approved by the ethical committee at the university where this research was conducted.

\section{Results}

\subsection{Manipulation check}

Repeated measures ANOVAs were conducted on pre- and postinduction positive and negative affect scores (with mood condition entered as a between-subject independent variable), to test whether the film clips were successful in inducing the intended moods. There was a significant interaction between mood condition and time, $F_{2,69}=4.58, p<.02$. Paired samples $t$-tests demonstrated that individuals in the negative mood induction had a significant decrease in positive affect after the induction $\left(t_{23}=3.53, p<.005\right)$, those in the neutral condition had a marginally significant decrease $\left(t_{23}=1.88, p<.08\right)$, and those in
Table 1

Mean positive and negative affect subscale scores (with standard error) from the PANAS before and after the mood induction (i.e., neutral, positive, or negative video clip).

\begin{tabular}{llllll}
\hline & Positive Affect & & & Negative Affect \\
\cline { 2 - 3 } \cline { 5 - 6 } \cline { 5 - 6 } & Pre-Induction & Post-Induction & & Pre-Induction & Post-Induction \\
\hline Neutral & $28.8(0.9)$ & $27.3(1.2)$ & & $15.2(1.0)$ & $13.2(0.7)$ \\
Positive & $29.2(0.9)$ & $29.4(1.0)$ & & $14.3(0.9)$ & $13.5(0.9)$ \\
Negative & $28.7(0.7)$ & $25.8(1.1)$ & & $13.3(0.6)$ & $16.5(1.1)$ \\
\hline
\end{tabular}

the positive condition had no significant change $\left(t_{23}=.45, n s\right)$. There was also a significant effect of mood condition on negative affect across time, $F_{2,69}=19.3, p<.001$, with paired-samples $t$-tests demonstrating that participants induced with a negative mood reported an increase in negative affect $\left(t_{23}=5.10, p<.001\right)$, those in the neutral induction reported a decrease $\left(t_{23}=2.97, p<.01\right)$, while those in the positive mood induction exhibited no significant change in negative affect $\left(t_{23}=1.41, n s\right)$. Participants' self-reported positive and negative affect pre- and post-induction are reported in Table 1.

Although the negative mood induction worked as expected, the positive mood induction was unsuccessful in altering either positive or negative affect, and the neutral mood induction paradoxically decreased negative affect as well as decreased (to a marginally significant extent) positive affect. As a result of the somewhat failed manipulation, the decision was made to re-assign participants to a low $(n=20)$, medium $(n=28)$ or high negative affect group $(n=24)$ on the basis of a tertiary split on post-induction negative affect scores (adapted from the approach taken by Herman, Polivy, Lank, \& Heatherton, 1988).

\subsection{State TSF}

An ANOVA was conducted in order to assess the state TSF-like experience, with the total State TSF score entered as a dependent variable, and the induction condition (TSF vs. neutral control) and participants' self-reported negative affect level (low, medium or high) entered as independent variables. A significant main effect of both TSF condition $\left[F_{1,66}=6.36, p<.02\right]$ and negative affect level emerged $\left[F_{2,66}=10.12, p<.001\right]$. These main effects were qualified by an interaction between TSF condition and negative affect level $\left[F_{2,66}=3.85, p<.03\right] .{ }^{1}$ Neuman-Keuls post-hoc tests demonstrated that only individuals with a medium level of negative affect were responsive to the TSF induction, as those induced with TSF reported significantly higher state TSF than did those exposed to the control induction $(p<.01)$. Those individuals who reported low levels of negative affect did not exhibit differences in state TSF scores across the induction conditions. Similarly, those with high negative affect also had statistically similar state TSF scores, regardless of whether they had been exposed to a TSF induction. Furthermore, those with high levels of negative affect who were exposed to the control induction reported significantly higher levels of state TSF than did those with low negative affect (control \& TSF induction), and those with medium negative affect (control condition), $p<.01$. Mean state TSF scores across negative affect subtypes are exhibited in Fig. 1.

To determine whether participants exposed to the TSF induction actually believed that they had gained weight as a result of imagining the food, participants' self-reported weight post-induction was compared with their self-reported weight at scheduling. However, no significant main effects emerged $\left(F^{\prime} s<1\right)$, nor was

\footnotetext{
1 The pattern of results stayed the same when the first two questions assessing negative affect (guilt and anxiety) were removed from the TSF state total score.
} 


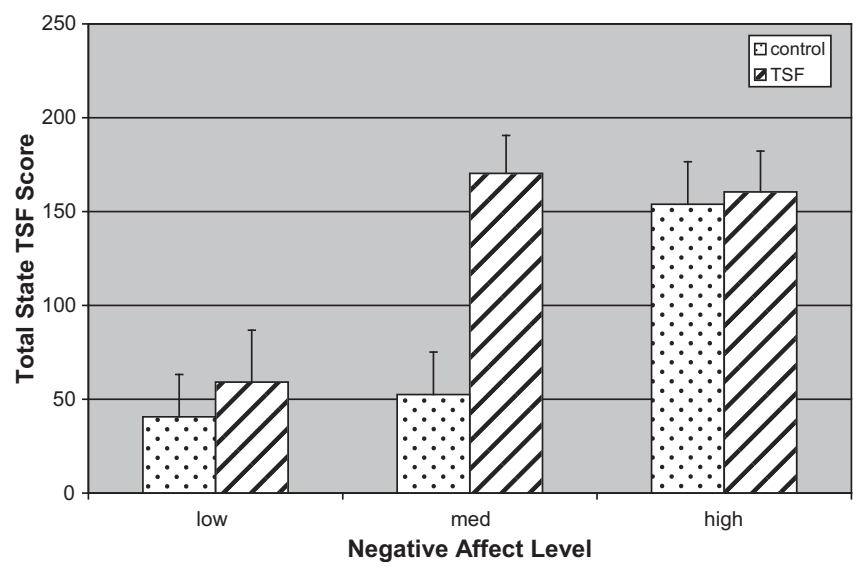

Fig. 1. State TSF scores (mean $+/$ - standard error) for individuals in the TSF induction condition (low negative affect $n=8$; medium negative affect $n=15$; high negative affect $n=13$ ), and neutral control condition (low negative affect $n=12$; medium negative affect $n=13$; high negative affect $n=11$ ).

there an interaction between TSF condition and negative affect level, $F_{2,65}=1.17, p>$. .

To ensure that (consistent with the findings of Study 1) no additional interaction existed with dietary restraint, the ANOVA on state TSF scores was re-conducted with restraint status entered as an additional independent variable. Due to low sample sizes (with $n=2-9$ per cell), the results must be interpreted with caution; however, restraint status did not interact with either negative affect level or TSF condition, nor was there a three-way interaction (all $F^{\prime} S<1$ ). Furthermore, controlling for restraint in the analyses (by including restraint scores as a covariate) did not change the pattern of results in the ANOVA on state TSF.

\subsection{Neutralization}

Likelihood ratio chi square analyses were performed to determine whether there was a significant effect of induction condition on the likelihood of neutralization. A significant effect of condition on neutralization emerged only for those high in negative affect $\left[\mathrm{L}^{2}(1, \mathrm{~N}=24)=4.04, p \leq .05\right]$, with $23 \%$ of those induced with TSF neutralizing while $0 \%$ of those in the control condition neutralized. As in Study 1, the neutralizers were exclusively restrained eaters (though small sample sizes prevented testing for significant differences based on dietary restraint status).

\subsection{Gift choice}

A significant effect of condition emerged on participants' choice of gift for those highest in negative affect $\left[\mathrm{L}^{2}(1, \mathrm{~N}=24)=4.14\right.$, $p<.05]$. High-negative-affect individuals in the control induction condition were more likely to choose a chocolate bar (63.6\%) than were those in the TSF condition (23\%). For those with low and medium levels of negative affect, participants were much less likely to choose a chocolate bar than an apple (20\% and $35.7 \%$ respectively chose a chocolate bar, and no significant differences across induction conditions emerged).

\subsection{Trait TSF}

No significant main effects of induction condition $\left(F_{1,63}=.36\right.$, $p>.55)$ or affect level $\left(F_{2,63}=2.68, p \leq .08\right)$ emerged on Trait TSF scores, nor was there a significant interaction between these factors $\left(F_{2,63}=1.82, p \leq .17\right)$.

\section{Discussion}

Due to an unsuccessful mood manipulation, in which the neutral mood induction led to significant changes in both positive and negative mood and the positive mood induction did not induce mood changes, participants were re-assigned to a mood condition based on their self-reported negative affect (low, medium, or high) after exposure to the mood manipulation. Based on this re-assignment, the results provided partial support for the original hypotheses. As expected, the TSF induction led to significant increases in state TSF, and the TSF induction interacted with level of negative affect. However, only medium levels of negative affect were associated with higher state TSF after TSF induction as compared to the control induction. It appeared that those with low levels of negative affect were protected against TSF, while those with high levels of negative affect appeared to exhibit high susceptibility to TSF-like experiences.

While those with high negative affect experienced a stronger susceptibility to a TSF-like experience (regardless of whether they received a control or TSF induction), only those who were exposed to the TSF induction were more likely to neutralize. This suggests that there is something particularly aversive about the TSF induction. Furthermore, those with high negative affect who were exposed to the control induction were more likely to choose the chocolate bar than were individuals in other conditions, while exposure to the TSF induction reduced the likelihood of choosing the chocolate bar. These differences between the neutralizing and food choice behavior of those high negative affect individuals (depending on their experimental induction condition) suggests there was some degree of increased susceptibility to the TSF induction relative to the control group.

The results suggest that medium levels of negative affect are associated with increased susceptibility to acute TSF inductions. Individuals with low negative affect appear to be protected against TSF, while those with high negative affect appear to experience TSF-like characteristics even after imagining neutral situations. The protective effect of low negative affect against state TSF is consistent with previous research, which demonstrated that positive affect leads to decreased estimation of body size (Kulbartz-Klatt et al., 1999). Similarly, the TSF susceptibility associated with high levels of negative affect is consistent with previous research which demonstrated a relationship between depressed mood and body size overestimation (McCabe, Ricciardelli, Sitaram, \& Mikhail, 2006; Taylor \& Cooper, 1986). Depressive symptoms lead individuals to focus selectively on negative aspects of themselves and their experiences (Beck, 2002). Furthermore, according to Beck, this negative outlook tends to be persistent, even in generally positive situations. Applying this theory to the current results, it may be that high levels of negative affect lead individuals to attend more to negative body information, even when imagining relatively neutral situations. While the direction of any possible causal link between depressive symptoms and eating pathology remains unclear, it does appear that negative affect can contribute to eating-disordered symptomatology (Polivy \& Herman, 2002).

As the current study used self-reported state negative affect to classify individuals into affective categories (low, medium, or high), it is not clear whether manipulation of both positive and negative affect would be more effective in influencing TSF-like experiences. Given the partial failure of the positive and neutral mood manipulations in the current study, it will be important to re-conduct this study using more effective inductions in order to gain further insight into this question. Studies which have used a combination of mood induction techniques (e.g., autobiographical memory plus music) appear to be successful in inducing positive and negative mood (e.g., Ingram, Bernet, \& McLaughlin, 1994; Kulbartz-Klatt et al., 1999), and could be considered for follow-up research. 
Despite the change in the manner of the TSF induction relative to Study 1 (i.e., the removal of the visual cue), restrained eaters appeared to be responsive to the induction. In fact, it was exclusively restrained eaters who exhibited neutralizing behavior after the TSF induction, providing some support for the notion that TSF is particularly aversive for those with higher weight/shape concerns. While limited sample size (and the exclusion of restraint status as an independent variable in Study 2) prevents firm conclusions to be drawn about the role of dietary restraint on TSF-like experiences, it appears that both restrained and unrestrained eaters can be induced with TSF, even in the absence of visual cues.

Although previous research demonstrated that both restrained and unrestrained eaters perceive a higher likelihood that they have gained weight after a TSF induction (relative to a control induction; Coelho et al., 2008), the current study demonstrated that participants did not actually report that they weighed more after the TSF induction (relative to their baseline self-reported weight). It is possible that the method used to assess perceived weight gain in the current study made it difficult to obtain effects in this nonclinical population - participants were informed that they would receive a small reward if they were accurate in their weight estimations. This offer of a reward was included to provide an opportunity for the experimenter to measure choice of healthy vs. unhealthy food; given that participants were already receiving either course credit or a cash voucher, it was thought that offering the food under the sole pretext of a "parting gift", as in previous research (e.g., Fishbach et al., 2003) might come across as suspicious. However, this offer of a reward for accurate weight reports may have dissipated possible distortions in perceived weight. Future research could investigate whether there are other more subtle, indirect ways of assessing perceived weight change. Furthermore, not all of the questions that have been used to assess TSF-like experiences are exclusive to this phenomenon. Guilt, anxiety, and desire to eat high-caloric foods may also be present after other types of manipulations. This suggestion is supported by the fact that individuals who were exposed to the control induction in both Studies 1 and 2 had some degree of a TSF-like experience (scores higher than 0). Future research investigating the assessment of TSF would provide further insight into the specific cognitions associated with this phenomenon.

\section{General conclusions}

The current series of studies demonstrated that 1) TSF-like experiences appear to be limited to thinking about eating highcaloric foods, and are not induced by mere exposure to food-related cues and 2) negative affect is associated with TSF-like experiences, such that individuals with low levels of negative affect appear to be protected against TSF, medium negative affect is associated with susceptibility to TSF inductions, and those with high negative affect are particularly vulnerable to TSF-like experiences, even when not specifically asked to think about eating fattening foods.

Overall, the research supports the notion that TSF is a valid phenomenon that is experienced (to at least some extent) by many females in the general population. The results also provide some initial evidence that negative affect is associated with TSF. This finding has clinical implications, as it suggests that negative affect may increase vulnerability to cognitive distortions. This, in turn, may interfere with treatment outcome. Indeed, researchers have demonstrated that individuals with bulimia who are high in negative affect have more eating pathology, greater distress, and are less likely to recover from their eating disorder (Stice, Bohon, Marti, \& Fischer, 2008). Along these lines, the integrative cognitive-affective therapy model was recently proposed, which postulates that negative affect mediates eating disorder symptoms and includes focus on modifying emotional experiences and behavioral tendencies in response to emotions as part of treatment for eating disorders (Wonderlich et al., 2008).

Non-eating-disordered overweight individuals who experience high levels of negative affect also have higher body-related concerns than do individuals with low levels of negative affect (Jansen, Havermans, Nederkoorn, \& Roefs, 2008). In conjunction with the findings of the current study, this research suggests that the role of negative affect in body concerns and eating pathology is applicable across the spectrum of eating-related concerns, from healthy, normal-weight individuals, restrained eaters, overweight, and eating-disordered individuals. Further research investigating the role of negative affect in susceptibility to body-related concerns and cognitive distortions such as TSF can ultimately provide insights into improving treatment and prevention of eating pathology.

\section{Acknowledgements}

This research was supported by a grant from the Canadian Institutes of Health Research awarded to the first author. The authors gratefully acknowledge Karolien van den Akker and Dian de Vries for their help with participant recruitment and data collection. Portions of this research were presented at the Swiss Winter Ingestion Conference, St. Moritz, Switzerland, March 2009.

\section{References}

Allison, D. B., Kalinsky, L. B., \& Gorman, B. S. (1992). A comparison of the psychometric properties of three measures of dietary restraint. Psychological Assessment, 4, 391-398.

Beck, A. T. (2002). Cognitive models of depression. In R. L. Leahy, \& E. T. Dowd (Eds.), Clinical advances in cognitive psychotherapy: Theory and application (pp. 29-61). New York: Springer Publishing Company, Inc.

Carter, F. A., Bulik, C. M., Lawson, R. H., Sullivan, P. F., \& Wilson, J. S. (1996). Effect of mood and food cues on body image in women with bulimia and controls. International Journal of Eating Disorders, 20, 65-76.

Coelho, J. S., Carter, J. C., McFarlane, T., \& Polivy, J. (2008). "Just looking at food makes me gain weight": experimental induction of thought-shape fusion in eating-disordered and non-eating-disordered women. Behaviour Research And Therapy, 46, 219-228.

Coelho, J. S., Jansen, A., Roefs, A., \& Nederkoorn, C. (2009). Eating behavior in response to food-cue exposure: examining the cue-reactivity and counteractive-control models. Psychology of Addictive Behaviors, 23, 131-139.

Fedoroff, I. C., Polivy, J., \& Herman, C. P. (1997). The effect of pre-exposure to food cues on the eating behavior of restrained and unrestrained eaters. Appetite, 28, 33-47.

Fett, A., Lattimore, P., Roefs, A., Geschwind, N., \& Jansen, A. (2009). Food cue exposure and body image satisfaction: the moderating role of BMI and dietary restraint. Body Image, 6, 14-18.

Fishbach, A., Friedman, R. S., \& Kruglanski, A. W. (2003). Leading us not unto temptation: momentary allurements elicit overriding goal activation. Journal of Personality and Social Psychology, 84, 296-309.

Forbush, K., \& Watson, D. (2006). Emotional inhibition and personality traits: a comparison of women with anorexia, bulimia, and normal controls. Annals of Clinical Psychiatry, 18, 115-121.

Geschwind, N., Roefs, A., Lattimore, P., Fett, A., \& Jansen, A. (2008). Dietary restraint moderates the effects of food exposure on women's body and weight satisfaction. Appetite, 51, 735-738.

Gonzalez, V. M., \& Vitousek, K. M. (2004). Feared food in dieting and non-dieting young women: a preliminary validation of the food phobia scale. Appetite, 43, 155-173.

Gross, J. J., \& Levenson, R. W. (1995). Emotion elicitation using films. Cognition $\mathcal{E}$ Emotion, 9, 87-108.

Hays, W. L. (1963). Statistics for psychologists. New York: Holt, Rinehart \& Winston.

Heilbrun, A. B., Jr., \& Flodin, A. (1989). Food cues and perceptual distortion of the female body: implications for food avoidance in the early dynamics of anorexia nervosa. Journal of Clinical Psychology, 45, 843-851.

Hepworth, R., Mogg, K., Brignell, C., \& Bradley, B. P. (2010). Negative mood increases selective attention to food cues and subjective appetite. Appetite, 54, 134-142.

Herman, C. P., Polivy, J., Lank, C. N., \& Heatherton, T. F. (1988). Anxiety, hunger and eating behavior. Journal of Abnormal Psychology, 96, 264-269.

Ingram, R. E., Bernet, C. Z., \& McLaughlin, S. C. (1994). Attentional allocation processes in individuals at risk for depression. Cognitive Therapy and Research 18, 317-332.

Jansen, A., Broekmate, J., \& Heymans, M. (1992). Cue exposure vs self-control in the treatment of binge eating: a pilot study. Behaviour Research And Therapy, 30 235-241. 
Jansen, A., Havermans, R., Nederkoorn, C., \& Roefs, A. (2008). Jolly fat or sad fat? subtyping non-eating-disordered overweight and obesity along an affect dimension. Appetite, 51, 635-640.

Kulbartz-Klatt, Y. J., Florin, I., \& Pook, M. (1999). Bulimia nervosa: mood changes do havean impact on body size estimation. British Journal of Clinical Psychology, 38, $279-287$.

Lafay, L., Thomas, F., Mennen, L., Charles, M. A., Eschwege, E., Borys, J., et al. (2001). Gender differences in the relation between food cravings and mood in an adult community: results from the Fleurbaix Laventie Ville Santé study. International Journal of Eating Disorders, 29, 195-204.

Legenbauer, T., Voëgle, C., \& Rüddel, H. (2004). Anticipatory effects of food exposure in women diagnosed with bulimia nervosa. Appetite, 42, 33-40.

McCabe, M. M., Ricciardelli, L. A., Sitaram, G., \& Mikhail, K. (2006). Accuracy of body size estimation: role of biopsychosocial variables. Body Image, 3, 163-171.

Nederkoorn, C., \& Jansen, J. (2002). Cue reactivity and regulation of food intake. Eating Behaviors, 3, 61-72.

Polivy, J., \& Herman, C. P. (2002). Causes of eating disorders. Annual Review of Psychology, 53, 187-213.

Polivy, J., Herman, C. P., \& Howard, K. (1988). The restraint scale: assessment of dieting. In M. Hersen, \& A. S. Bellack (Eds.), Dictionary of behavioral assessment techniques (pp. 377-380). New York: Pergamon Press.

Radomsky, A. S., de Silva, P., Todd, G., Treasure, J., \& Murphy, T. (2002). Thoughtshape fusion in anorexia nervosa: an experimental investigation. Behaviour Research And Therapy, 40, 1169-1177.
Shafran, R., \& Robinson, P. (2004). Thought-shape fusion in eating disorders. British Journal of Clinical Psychology, 43, 399-407.

Shafran, R., Teachman, B. A., Kerry, S., \& Rachman, S. (1999). A cognitive distortion associated with eating disorders: thought-shape fusion. British Journal of Clinical Psychology, 38, 167-179.

Soetens, B., Braet, C., van Vlierberghe, L., \& Roets, A. (2008). Resisting temptation: effects of exposure to a forbidden food on eating behaviour. Appetite, 51, 202-205.

Stice, E., Bohon, C., Marti, C. N., \& Fischer, K. (2008). Subtyping women with bulimia nervosa along dietary and negative affect dimensions: further evidence of reliability and validity. Journal of Consulting and Clinical Psychology, 76, 1022-1033.

Taylor, M. J., \& Cooper, P. J. (1986). Body size overestimation and depressed mood. British Journal of Clinical Psychology, 25, 153-154.

Taylor, M. J., \& Cooper, P. J. (1992). An experimental study on the effect of mood on body size perception. Behaviour Research And Therapy, 30, $53-58$.

Watson, D., Clark, L. A., \& Tellegen, A. (1988). Development and validation of brief measures of positive and negative affect: the PANAS scales. Journal of Personality and Social Psychology, 54(6), 1063-1070.

Wonderlich, S. A., Engel, S. G., Peterson, C. B., Robinson, M. D., Crosby, R. D., Mitchell, J. E., et al. (2008). Examining the conceptual model of integrative cognitive-affective therapy for BN: two assessment studies. International Journal of Eating Disorders, 41, 748-754. 